\title{
Forelimb joints contribute to locomotor performance in reindeer (Rangifer tarandus) by maintaining stability and storing energy
}

Guoyu Li ${ }^{1}$, Rui Zhang ${ }^{\text {Corresp., } 1}{ }^{1}$, Dianlei Han ${ }^{1}$, Hao Pang ${ }^{1}$, Guolong Yu ${ }^{1}$, Qingqiu Cao ${ }^{1}$, Chen Wang ${ }^{1}$, Lingxi Kong ${ }^{1}$, Wang Chengjin ${ }^{1}$, Wenchao Dong ${ }^{1}$, Tao Li ${ }^{1}$, Jianqiao $\mathbf{L i}^{1}$

${ }^{1}$ Key Laboratory of Bionic Engineering, Ministry of Education, Jilin University, Changchun, People's Republic of China

Corresponding Author: Rui Zhang

Email address: zhangrui@jlu.edu.cn

Reindeer (Rangifer tarandus) have lengthy seasonal migrations on land and their feet possess excellent locomotor characteristics that can adapt to complex terrains. In this study, the kinematics and vertical ground reaction force (GRF) of reindeer forelimb joints (interphalangeal joint $b$, metacarpophalangeal joint $c$, and wrist joint $d$ ) under walk, trot 1 , and trot 2 were measured using a motion tracking system and Footscan pressure plates. Significant differences among different locomotor activities were observed in the joint angles, but not in changes of the joint angles $\left(\alpha_{b}, \alpha_{c}, \alpha_{d}\right)$ during the stance phase. Peak vertical GRF increased as locomotor speed increased. Net joint moment, power, and work at the forelimb joints were calculated via inverse dynamics. The peak joint moment and net joint power related to the vertical GRF increased as locomotor speed increased. The feet absorbed and generated more energy at the joints. During different locomotor activities, the contribution of work of the forelimbs changed with both gait and speed. In the stance phase, the metacarpophalangeal joint absorbed more energy than the other two joints while trotting and thus performed better in elastic energy storage. The joint angles changed very little $\left(\sim 5^{\circ}\right)$ from 0 to $75 \%$ of the stance phase, which reflected the stability of reindeer wrist joints. Compared to typical ungulates, reindeer toe joints are more stable and the stability and energy storage of forelimb joints contribute to locomotor performance in reindeer. 
1 Forelimb joints contribute to locomotor performance in

5 Guoyu Li ${ }^{1}$, Rui Zhang*1, Dianlei Han ${ }^{1}$, Hao Pang ${ }^{1}$, Guolong Yuㄹ, Qingqiu Cao ${ }^{1}$, Chen Wang

8 Key Laboratory of Bionic Engineering, Ministry of Education, Jilin University, Changchun, 


\section{Abstract}

16 Reindeer (Rangifer tarandus) have lengthy seasonal migrations on land and their feet possess

17 excellent locomotor characteristics that can adapt to complex terrains. In this study, the 18 kinematics and vertical ground reaction force (GRF) of reindeer forelimb joints (interphalangeal 19 joint $b$, metacarpophalangeal joint $c$, and wrist joint $d$ ) under walk, trot 1 , and trot 2 were 20 measured using a motion tracking system and Footscan pressure plates. Significant differences 21 among different locomotor activities were observed in the joint angles, but not in changes of the 22 joint angles $\left(\alpha_{\mathrm{b}}, \alpha_{\mathrm{c}}, \alpha_{\mathrm{d}}\right)$ during the stance phase. Peak vertical GRF increased as locomotor speed 23 increased. Net joint moment, power, and work at the forelimb joints were calculated via inverse 24 dynamics. The peak joint moment and net joint power related to the vertical GRF increased as 25 locomotor speed increased. The feet absorbed and generated more energy at the joints. During 26 different locomotor activities, the contribution of work of the forelimbs changed with both gait 27 and speed. In the stance phase, the metacarpophalangeal joint absorbed more energy than the 28 other two joints while trotting and thus performed better in elastic energy storage. The joint 29 angles changed very little $\left(\sim 5^{\circ}\right)$ from 0 to $75 \%$ of the stance phase, which reflected the stability 30 of reindeer wrist joints. Compared to typical ungulates, reindeer toe joints are more stable and 31 the stability and energy storage of forelimb joints contribute to locomotor performance in 32 reindeer.

34 Keywords: reindeer, locomotion, biomechanics, inverse dynamics, joint function. 


\section{Introduction}

38 Large animals like ungulates and humans exhibit better locomotor efficiency than small animals 39 like mice [1,2]. This is because large animals use the ground reaction force (GRF) to store 40 mechanical energy in their elastic feet to drive the trunk forward in a locomotor cycle. The 41 storage and generation of elastic energy in the feet is an efficient way to reduce metabolic energy 42 cost during locomotion [3]. Stretching of compliant tendons also allows limb muscles to save 43 energy by isometric contraction under load [4]. Thus, the muscle-tendon units of foot joints in 44 large animals serve important functions in energy storage, stabilization, and shock absorption.

45 In horses, during different locomotor activities, such as walking and trotting, the long digital 46 flexor tendons stretch and recoil from metacarpophalangeal (MCP) dorsiflexion and 47 plantarflexion, leading to elastic energy storage and energy generation at the joints [5]. This 48 action is like a passive spring and benefits the forelimbs during locomotion [6]. The MCP joints 49 are controlled by long tendons, superficial and deep digital flexor tendons, accessory ligaments, 50 and muscles. These tendons are ideal structures for energy storage and generation [7].

51 The stability of foot joints is associated with animal locomotion. For a trotting horse, both the 52 elbows and shoulders of the forelimbs have net extension moments, but there is little joint 53 movement when the moments are maximized. These joints are relatively rigid, which allow the 54 trunk muscles to absorb and transmit energy [8]. The soft tissues at animal joints have important 55 functions during locomotion. Additionally, kinematic parameters, such as joint angle, joint speed, 56 and plantar pressure, all differ depending on locomotor speed and gait $[9,10]$.

57 Gait refers to the pattern and order of limb locomotion. Most animals use different gaits based on 58 their terrain and locomotor speeds [11,12]. Gaits are categorized by order of ground contact into 59 walking, trotting, and galloping [13]. Quadrupeds use different gaits depending on their locomotor speeds and select walking, trotting, or galloping during low-, moderate-, and highspeed locomotion activities, respectively. While walking and trotting, the limbs are in symmetrical gaits, and the left and right limbs are almost under constant relative phases with at least one limb in the stance phase. While galloping, the limbs are in asymmetrical gaits. As the left and right limbs change the relative phase with locomotor speed, a swing phase exists in which the four limbs are simultaneously in the air [14]. Studies on the gaits of horses, deer, and 
67 mechanism of gait selection corresponding to locomotor speed is related to animal balance, 68 speed, and energy saving [15-19].

69 Based on locomotion speed and GRF, researchers have used various methods for studying the 70 dynamics of animal limbs in detail. Pandy et al. calculated the inter-articular force, joint moment, 71 and power of goats during different locomotion activities by using GRF and limb movement, and 72 found that the foot inertia was small and negligible relative to the trunk inertia [11]. Dutto et al. 73 measured the GRF, joint angle, moment, and power while trotting, and analyzed the kinematics 74 and dynamics of the four limbs, as well as the energy storage and consumption of tendons 75 [20,21]. Moreover, the muscle stress of horse limbs while galloping was 200-400 kpa, and long 76 tendons and extremely short pinnate muscle fibers allowed force production to be economical 77 and enhanced the storage of tendon elastic energy [22].

78 Load bearing and locomotion differ between the forelimbs and hindlimbs of animals. While 79 trotting, the maximum vertical GRFs in the forelimbs and hindlimbs of German Shepherds were $80 \sim 63 \%$ and $\sim 37 \%$ of their body weight, respectively, and the impact on the forelimbs was 81 significant [23]. While walking and trotting, the maximum vertical GRFs of the forelimbs were $82 \sim 1.7$ and $\sim 1.4$-times those of the hindlimbs, respectively [24]. The functions of horse forelimbs 83 also differ depending on the GRFs, as the forelimbs mainly exert a braking effect and decrease 84 the speed and kinetic energy, while the hindlimbs mainly play propulsive roles [25,26]. While 85 86 trotting, the maximum vertical GRFs of the forelimbs and hindlimbs are $\sim 10$-times the horizontal reaction and lateral reaction forces, while the vertical GRFs are much larger than the other component forces [26]. The GRFs of limbs in walking cows and gibbons are the same [27,28]. Reindeer, a typical Arctic migratory animal, have a limb structure suitable for migration in complex environments [29]. They can adapt to various terrains, such as ice, snow, wetland, and sand [30]. Reindeer seasonally migrate long distances on land and some populations migrate farther than other terrestrial mammals [31]. Additionally, all fibers in reindeer skeletal muscles have a high oxidative capacity, which may be related to endurance activity [32]. The sizes and structures of foot soles differ between forelimbs and hindlimbs [33]. The foot soles of the 94 forelimbs are longer than the hindlimbs $(87.0 \pm 1.6$ vs. $74.6 \pm 1.0 \mathrm{~cm})$. We speculate that this 95 difference may be attributed to the different functions between reindeer forelimbs and hindlimbs during long migrations. 
97 The toe and wrist joints of reindeer forelimbs are more stable than typical ungulates, and the

98 stability of the wrist joint is higher. Reindeer MCP joints play the same energy storage role as in 99 typical ungulates. Additionally, the work contribution from the forelimbs changed with gait and 100 speed. In this study, the plantar pressure, kinematics, net joint power, and locomotor strategy of 101 reindeer forelimbs were investigated using the vertical GRFs and limb movements during 102 different locomotion activities. Based on previous studies, we used inverse dynamics and the 103 static approach to explore the functions of the main forelimb joints, including energy saving and 104 stabilization. We investigated whether the functions of interphalangeal joint $b, \mathrm{MCP}$ joint $c$, and 105 wrist joint $d$ in reindeer forelimbs were related to energy saving and stabilization during different

106

107 108

109

110 111

\section{Materials and Methods}

\section{Samples}

114 Fifteen healthy 8-year-old adult reindeer, including seven males and eight females, were selected 115 from the Evenki ethnic group in Genhe City, China. The female reindeer were all excluded to 116 rule out sex differences. Three males rushed to the fence and suffered foot injuries during 117 training, and thus were not used in the experiment. Finally, four other easily-trained and healthy 118 male reindeer were selected as experimental subjects. The animal laboratory of Jilin University 119 has granted ethical approval (No. 3130068) to this experiment. And the field experiments were 120 approved by the Breeding Garden of Reindeer. The masses, shoulder widths, and body lengths of 121 the subjects were $118.75 \pm 14.93 \mathrm{~kg}, 1.22 \pm 0.51 \mathrm{~m}$, and $1.89 \pm 0.83 \mathrm{~m}$, respectively (mean \pm standard 122 deviation). All subjects were in healthy condition and had not undergone any surgical treatment 123 or other invasive procedures. The reindeer were kept in an outdoor fenced area $\left(2 \times 10^{3} \mathrm{~m}^{2}\right) \mathrm{during}$ 124 the day with adequate supply of food and water, which was close to a semi-wild state, and were 125 released into a wild forest at night. Before data collection, each reindeer was trained to walk and 126 trot on the runway, for no less than 30 minutes, twice a day for one month. Four right forelimbs 
127 of naturally dead reindeer were purchased and amputated from the wrist joints. The lower half 128 of each forelimb was saved and sent for CT scans.

\section{Preparing the field}

130 The field was $80 \mathrm{~m}$ long surrounded by 1.5 -m-high fences with a $3-\mathrm{m}$-long and $1.5-\mathrm{m}$-wide data 131 acquisition area in the middle (Fig. 1). The outside of the data acquisition area was a 77-m-long 132 and 2-m-wide hard ground runway. The stones, weeds, and other debris were removed to ensure 133 that the runway was even and there were places saved for reindeer to rest and eat at both ends. A 134 pressure plate $\left(2096 \times 472 \mathrm{~mm}^{2}, 500 \mathrm{~Hz}\right.$ sampling, 16384 sensors with $0.5 \times 0.7 \mathrm{~cm}^{2}$, USBII 135 interface; Olen, Belgium) was placed on the runway and its position was adjusted to ensure that 136 it was on the same plane as the runway. The pressure plate was connected via a signal 137 conditioner (National Instruments, Austin TX, USA) to a computer (Dell, Xiamen, China) to 138 record the data. One camera was placed on one side of the data acquisition area and another two 139 cameras were placed on the other side. A high-speed camera system involving three synchronous 140 digital cameras (Casio Exilim EX-FH25, Tokyo, Japan; 120 frame $\cdot \mathrm{s}^{-1}$ ) was established. Prior to 141 the experiment, a 36-point, three-dimensional calibration frame, located in the plane of 142 movement over the force platform, was recorded for calibration.

143 During the procedures, the feeder used food or training instructions to guide the reindeer to walk 144 and trot steadily on the runway. Adequate rest and food were provided for the animals within this 145 period to prevent fluctuations in the test data. The locomotion of reindeer was divided by the 146 gaits and speeds into walk $(\mathrm{u}=0.44 \pm 0.08)$, trot $1(\mathrm{u}=0.95 \pm 0.15)$, and trot $2(\mathrm{u}=1.46 \pm 0.24)$.

\section{Markers and joint angles}

148 We tested the three-dimensional (3D) coordinates of the five joints ( $a, b, c, d, e$ (Fig. 2A)) in the 149 right forelimbs and three joint angles $\left(\alpha_{\mathrm{b}}, \alpha_{\mathrm{c}}, \alpha_{\mathrm{d}}\right)$ by using a three-camera motion tracking system 150 (Simi Motion 2D/3D ${ }^{\circledR} 7.5$ software, SIMI Reality Motion Systems GmbH, Germany). The right 151 forelimbs of the four adult reindeer underwent CT scans, and a 3D geometric model (Fig. 2C) of 152 metacarpal, the second, third, fourth and fifth digits was established. Markers $a$ (the dorsal of the 153 hoof at the third digit), $b$ (the proximal phalanx and the middle phalanx of the third digit at the 154 joint), $c$ (MCP joint), and $d$ (wrist joint) were located according to the 3D limb model. The 155 location of $e$ (elbow joint) was determined based on the joint anatomy. 
156 Regular circular reflective stickers $(\mathrm{R}=1.5 \mathrm{~cm})$ were used as markers which were attached to the 157 main joints of their right forelimbs (Fig. 2A). Researchers found that the relative locations of the 158 distal phalanx and the middle phalanx were almost on one straight line [34]. Since the distal 159 phalanx inside the hoof was hard to measure, the hoof and distal phalanx were taken as one part ( 160 thepart surrounded by dotted lines in Fig. 2C) and marker $a$ on the hoof was considered as the 161 joint of the middle phalanx and the distal phalanx.

162 We defined three joint angles (Fig. 2E), including the joint angles between the middle phalanx 163 and the proximal phalanx $\left(\alpha_{\mathrm{b}}\right)$, between the proximal phalanx of the third digit and the 164 metacarpal $\left(\alpha_{\mathrm{c}}\right)$, and between the metacarpal and radius $\left(\alpha_{\mathrm{d}}\right)$.

\section{Vertical GRF}

166 The vertical GRF of each right forelimb was measured by the pressure plate. Before the 167 measurement, the subject's weight was input into the computer and then the subject moved on 168 the pressure plate to complete calibration. The pressure data for the right forelimb were collected 169 and analyzed, using Footscan 7Gait 2nd generation (RSscan International, Oren, Belgium). 170 Footscan was used to export the fore-aft coordinates of the COP during the full stance phase 171 duration. We calculated the average path of the centre of pressure (COPy; fore-aft component) 172 for a series of forefoot sequences within the same speed range. In terms of the kinematic data, 173 when the displacement of the ungula cusps on Z-axis changed from a negative value to 0 , it was 174 identified as the touch-down moment of pressure plate data. When the displacement of the 175 ungula cusps on Z-axis changed from 0 to a positive value, it was identified as the lift-off 176 moment of pressure plate data (Fig. 2). Taking the ungula cusps as the origin, the coordinates of

177 the COP and the joints relative to the ungula cusp were calculated, and then a global coordinates 178 of the kinematic data and the COP was established. The relationship between GRF and time was 179 drawn and normalized to the sample mass. Angular velocity, stance phase, vertical GRF, net 180 joint moment, power, and work were calculated on Origin Pro 2015 (OriginLab Corporation, 181 Northampton, MA, USA) based on the data from the joint 3D coordinates.

\section{Net joint moment}

183 The mass of the animal foot is small and the toe joints (the joint of the middle phalanx and the 184 distal phalanx, the joint of the proximal phalanx and the middle phalanx) and wrist joint during 185 the stance phase displaced less than other proximal joints [35,36]. Therefore, we applied a static 
186 approach regardless of gravity and inertia. The net joint moment $\left(M_{\mathrm{m}}\right)$ was determined by 187 vertical GRF and joint position (Fig. 2D) and was equal to the product of vertical GRF (averaged 188 from the four reindeer at walk, trot 1, and trot 2) and $L$ (vertical distance vector from the joint 189 marker to the GRF) [37]:

$$
M_{\mathrm{m}}=G R F \cdot L
$$

191 We have defined the positive direction of the forelimb joint moment of the reindeer (Fig. 2D):

192 - For the wrist joint $(d)$, net extension moment is positive (produced by extensor muscle), and net 193 flexion moment is negative (produced by flexor muscle);

194 - For the joints of the toes $(b, c)$, net flexion moment is positive (produced by the plantar flexor 195 muscle) and net extension moment is negative (produced by the plantar extensor muscle).

196 Net joint power and work

197 To estimate the energy absorbed and generated by the interphalangeal joint $b$, the MCP joint $c$, 198 and the wrist joint $d$, we calculated the net joint power. Joint angular velocity was calculated 199 from the joint angle versus the time derivative by using a differential function (the central 200 difference method). The positive direction of angular velocity is the same as that of the joint 201 moment (Fig. 2E). The net joint power $\left(P_{\mathrm{m})}\right.$ of the joint equals the product of net joint moment $202\left(M_{\mathrm{m}}\right)$ and joint angular velocity $(\omega)$, where $\omega$ is averaged from the four subjects at walk, trot 1 , 203 and trot $2[35]$ :

$$
P_{\mathrm{m}}=M_{\mathrm{m}} \cdot \omega(2)
$$

205

206

207

208

209

210

211

212

213

When the directions of the joint moment and joint angular velocity are the same, the net joint power is positive, and otherwise it is negative. Positive work and negative work represent the energy generated and absorbed by muscle-tendon units respectively [38].

\section{Gait and speed}

Each reindeer completed at least five groups of tests (walk, trot 1, and trot 2) on the hard ground. We combined the research on the gaits of other animals (e.g. goats and horses [12,19]) and reindeer's locomotor postures and then sorted out the gaits and order of the footprints (Fig. 1B). The reindeer's postures of the right forelimbs at walk, trot 1, and trot 2 during the stance phase were shown in Fig. 3. The moments of touch-down, mid-stance, and lift-off are $0 \%, 50 \%$ and $100 \%$ of the stance phase respectively. 
215 - Walk: Symmetrical gait. At any time during the stance phase, at least two limbs are on the

216 ground and four limbs leave the ground in sequence (e.g. the leaving sequence of left rear - left

217 front - right rear - right front) (Fig. 2B).

218 - Trot: Symmetrical gait. Each forelimb and its diagonal hindlimb move in the same phase, and

219 only two limbs are in the stance phase (sometimes all four legs are in the swing phase at the 220 same time, e.g. the leaving sequence of left rear and right front-right rear and left front) (Fig. 2B).

221 Speed data were normalized by Froude number $(u)$, where $v$ is the average velocity, $l$ is the

222 height of the shoulder joint from touch-down to lift-off, and $g$ is the acceleration of gravity:

223

$$
u=\frac{v}{\sqrt{g \cdot l}}
$$

224 In order to examine changes with speed, relevant variables from all trotting trials were divided 225 into two bins indicative of trot 1 (range of $u$ : 0.8-1.1) and trot 2 (range of $u$ : 1.1-1.7). The speeds 226 of reindeer at walk, trot 1 , and trot 2 , after nondimensionalization, are $0.44 \pm 0.08,0.95 \pm 0.15$, and $2271.46 \pm 0.24$ (mean \pm S.D.).

\section{Statistical analysis}

229 Vertical GRF, net joint moment and net joint work were normalized to stance duration and to 230 reindeer's mass. During walk, trot 1, and trot 2 in the stance phase, the kinematics and the 231 plantar pressure data of four reindeer were combined to analyze the temporal variation of joint 232 angles and vertical GRFs during the stance phase. The means and the variances of the vertical 233 distance vector from the joint marker to the GRF $(L)$ and joint angular velocity $(\omega)$ were 234 obtained from four samples at walk, trot 1 , and trot 2, respectively. The joint angular velocity 235 was the derivative of the joint angle with respect to time. Meanwhile, the means of the vertical 236 GRF from four reindeer were obtained. Based on the above data, the net joint moment and the 237 net joint power were calculated by using the formula. The work done at the joint was the integral 238 of the net joint power with respect to time. Statistical analyses were conducted to examine the differences in different gaits and speeds, three key indicators (joint angles at touch-down, midstance and lift-off) between slow walking and trotting gaits/ trot 1 and trot 2 speeds by using

241 Origin Pro 2015 (OriginLab Corporation, Northampton, MA, USA). We used one-way ANOVA 242 statistical technique to analyze the effect of locomotor gait and speed on each joint angle 243 indicator. $F$-test was conducted to examine whether these two variations are significantly 244 different. Statistical significance level was considered as $\mathrm{P}<0.05$. 


\section{Results}

\section{Joint angles}

248 During walk, trot 1, and trot 2 in the stance phase, the reindeer interphalangeal joint angle $\alpha_{\mathrm{b}}$ (Fig. 249 4A-C), MCP joint angle $\alpha_{\mathrm{c}}$ (Fig. 4D-F), and wrist joint angle $\alpha_{\mathrm{d}}$ (Fig. 4G-I) showed similar 250 patterns and ranges. Therefore, the data of joint angles of the four reindeer were combined to 251 analyze the temporal variation of joint angles during the stance phase. The stick figure of the 252 forelimbs during different locomotor stance phases was shown in Fig. 4J-L. At the moment of 253 touch-down, the limb joints $(b, c, d)$ first moved toward the ground and then left the ground after 254 touching the lowest point. This pattern of motion may be related to energy saving.

255 The integrated data correspond to the means and variances of $\alpha_{b}, \alpha_{c}$ and $\alpha_{d}$ (Fig. 5A-C) at walk, 256 trot 1 , and trot 2 in the stance phases. The joint angles $\left(\alpha_{b}, \alpha_{c}, \alpha_{d}\right)$ displayed similar patterns 257 during different locomotor stance phases. The $\alpha_{\mathrm{b}}$ increased (joint plantarflexion) during the early 258 stance phase (about 0-30\%), decreased (joint dorsiflexion) in the mid-stance phase (about 30\%$25980 \%$ ), and rose (joint plantarflexion) in the late stance phase (about 80\%-100\%). The 260 interphalangeal joint $b$ plantarflexed in the late stance phase, and the hoof gradually lifted off the 261 ground with the tip still in contact with the ground.

262 During different activities, the maximum and minimum values of $\alpha_{\mathrm{b}}$ and $\alpha_{\mathrm{c}}$ during the stance 263 phase and the corresponding time points differed. Also the joint range of motion (ROM) was 264 larger at the trotting gaits than at the walking gait. The ROMs of $\alpha_{\mathrm{d}}$ among the three joint angles 265 were the greatest, around $29^{\circ}, 30^{\circ}$ and $35^{\circ}$ during walk, trot 1 and trot 2 , respectively. The 266 ROMs of $\alpha_{\mathrm{b}}$ were the smallest, around $26^{\circ}, 27^{\circ}$ and $33^{\circ}$ during walk, trot 1 and trot 2 , 267 respectively. Therefore, the relationships of joint angles with gaits and speeds showed that the 268 joints can adapt to different gaits and locomotor speeds.

\section{Vertical GRF}

270 The forelimbs have different vertical GRFs at different time points during the stance phase.

271 According to the time corresponding to the peak vertical GRF, the forelimb locomotion can be 272 divided into a braking phase and a propulsive phase [39]. The vertical GRF increased with time 273 during the braking phase, and decreased with time during the propulsive phase (Fig. 6). 
274 The peak vertical GRFs (normalized to body mass) during walk, trot 1, and trot 2 were 8.95, 275 11.33, and 12.80 times the body mass, respectively, and the corresponding peak time was $27657.03 \%, 50.45 \%$, and $47.78 \%$ of the stance phase, respectively. The gaits and locomotor speeds 277 of reindeer affect the vertical GRF. As for different gaits, the peak vertical GRF at trot was larger 278 than that at walk. At the same gait, the peak vertical GRF at trot 2 was larger than that at trot 1.

\section{Net joint moment}

280 In different activities, the forelimb joints $b$ and $c$ of reindeer in the stance phase (about 0 to $100 \%$ ) 281 produced positive net flexion moment by the plantar flexor (Fig. 7). Joint $d$ in the early stance 282 phase (about 0 to $75 \%$ ) and late stance phase (about 75 to 100\%) generated the negative net 283 flexion moment and positive net extension moment respectively by the flexor and extensor 284 muscles (Fig. 8). Reindeer and horses have similar net joint moment curves for joints $c$ and $d$ 285 when trotting on hard ground [20].

286 In different activities, reindeer have different peak net joint moments at the forelimb joints. 287 Joints $b, c$, and $d$ reached the peak net joint moments at about $45 \%, 50 \%$ and $30 \%$ of the stance 288 phase, respectively. The peak net joint moments at walk, trot 1 , and trot 2 were $0.28,0.37$, and $2890.42 \mathrm{Nm} \cdot \mathrm{kg}^{-1}$ at joint $b, 0.55,0.79$, and $0.93 \mathrm{Nm} \cdot \mathrm{kg}^{-1}$ at joint $c$, and $-0.95,-1.35$, and -1.78 $290 \mathrm{Nm} \cdot \mathrm{kg}^{-1}$, at joint $d$, respectively. The vertical GRF of reindeer forelimbs increased with the 291 rising locomotor speed and, accordingly, the peak joint moment also rose. Since the vertical 292 distance vector of vertical GRF from joint $d$ was the largest, joint $d$ had the greatest peak joint 293 moment, followed successively by joint $c$ and joint $b$.

\section{Net joint power and work}

295 The net joint moment reflects the activity (extension and flexion) of muscles (extensors and 296 flexors), but not the changes of energy in the muscle-tendon units at the joints. Net joint power 297 and work, which are directly related to the energy absorption and generation at the limb joints 298 [40], at the forelimb joints were shown in Figs. 7 and 8. As mentioned above, the net joint 299 moment and angular velocity at the forelimb joints increased with the rise of locomotor speed. 300 Therefore, the net joint power at the joints increased accordingly. The net joint power ranges at 301 joint $c$ were the largest and were -0.37 to $0.06,-0.19$ to 0.21 , and -4.37 to $2.46 \mathrm{~W} \cdot \mathrm{kg}^{-1}$ at walk, 302 trot 1, and trot 2, respectively. As the locomotor speed was accelerated, the net joint power range 303 was enlarged and thus the feet need to absorb and generate more energy at the joints. 
304 In different activities, reindeer had similar work patterns at the same joint. From about 0 to $55 \%$

305 of the stance phase, the dorsiflexion of joint $c$ produced a net flexion moment and the foot 306 absorbed energy at the joint. From about 55\% to $100 \%$ of the stance phase, joint $c$ plantarflexed 307 and the plantar flexor and extensor muscles generated and absorbed energy, respectively (details 308 of energy absorption and generation at each joint are shown in Table 1). The energy changes at 309 the limb joints are related to joint functions, such as energy storage and stabilization [41,42].

\section{Discussion}

313 We investigated whether the functions of interphalangeal joint $b, \mathrm{MCP}$ joint $c$, and wrist joint $d$ 314 in the forelimbs correlated with energy saving and stability. Depending on the locomotor 315 postures and speeds, reindeer locomotor activities were divided into walk, trot 1, or trot 2 . In 316 different locomotion activities, we measured the temporal changes of plantar pressure and joint 317 angles in the right forelimbs of four healthy adult male reindeer. Based on inverse dynamics, we 318 calculated the net joint moment and net joint power of the right forelimbs, and the energy 319 absorption and generation by the limb joints.

320 Locomotor strategies change depending on the speed and gait adopted by the animal. For 321 example, goat limbs adjust the work of muscles and tendons to adapt to a walk or trot locomotion 322 on slope-variable surfaces [43]. Horses rely on the minimization of metabolic costs and change 323 gaits based on a range of different locomotion speeds, including the most energy-efficient trot 324 gait [18]. In our study, during different gaits (walk or trot) and speeds (trot 1 or trot 2), 325 significant differences were detected in the reindeer joint angles at the moments of touch-down, 326 mid-stance, and lift-off (Fig. 9), which may be associated with reindeer locomotor strategies.

327 Although reindeer changed locomotor strategies during different gaits and speeds, we still found 328 some similarities, such as the elastic energy saving function of joint $b$ and the effect of joint $d$.

\section{Contribution of work change with gaits and speeds}

330 Most animals use the inverted pendulum model in their walking gaits and restore mechanical 331 energy via the periodic conversion of kinetic and potential energies $[44,45]$. While trotting, the 332 spring-mass system and inverted pendulum model are used, wherein the limbs act as springs that 333 store and generate energy, which is characterized by a significant reduction in the difference 334 between the potential and kinetic energies during the stance phase [46,47]. In our study, 
335 significant differences were detected in joint angles $\alpha_{b}$ and $\alpha_{c}$ between walking and trotting gaits, 336 and in $\alpha_{b}$ and $\alpha_{d}$ between trot 1 and trot 2 (Fig. 9). In the trotting gait, the MCP joint absorbed 337 more energy than the other two joints (Table 1), but in the walking gait, the MCP joint absorbed 338 less energy than the wrist joint. This may be attributed to the preference of animals over the 339 inverted pendulum gait at low speeds and over the mass spring inverted gait at high speeds, 340 which both enhanced locomotor performance and energy saving [48,49]. The different motion 341 patterns among different gaits and speeds in reindeer forelimbs may be caused by the more 342 efficient energy mechanism.

343 Reindeer have enhanced MCP joints. While walking, the proximal phalanx pivots about joint $b$ 344 (stance phase of 0-10\%) with slight downward and upward movements (Fig. 5A). However, 345 while trotting, the distal phalanx moves downward (joint $b$ plantarflexion) for a prolonged period 346 of time (stance phase of 0-20\%) (Fig. 5A). Owing to the stretching and recoiling of plantar

347 flexor tendons, plantarflexion and dorsiflexion of interphalangeal joint $b$ are typical in loading 348 and rebounding patterns. This indicates that the elastic elements at the toe joints offset the GRF 349 and the trotting gait can reduce pressure, as well as protect the soft tissues of the toes by 350 prolonging the foot-to-ground contact time ratio.

351 As previously reported in horses, as locomotor speed increased, the positive and negative work 352 done by MCP joint $c$ increased significantly, and elastic energy storage and generation also 353 increased [20]. This finding is consistent with our results. When the ROM of MCP joints and net

354 flexion moment increased as locomotor speed increased, the foot work of reindeer at the joint 355 also increased. The difference in the ROM of wrist joint $d$ was small among different activities $356\left(\sim 5^{\circ}\right)$, but as locomotor speed increased, the vertical GRF and angular velocity also increased, 357 thereby increasing the net joint moment and net joint power. Compared to the trotting gait, the 358 power of the wrist joint was smaller, but fluctuated more severely in the walking gait (Fig. 8A). 359 Thus, slow locomotor activities may require a higher level of neural control [50].

\section{Comparison of reindeer forelimb joints to typical ungulates}

361 Ungulate locomotion has evolved in vastly different patterns depending on the specific habitat of 362 a given species [51]. Compared to horse forelimbs, reindeer toe joints are stable, as the ROMs 363 between the interphalangeal and MCP joints are smaller. The ROMs of interphalangeal joint $b$ in 364 reindeer and horses are $\sim 30^{\circ}$ and $\sim 40^{\circ}$, respectively, and the ROMs of MCP joint $c$ in reindeer 365 and horses are $\sim 31^{\circ}$ and $\sim 40^{\circ}$, respectively [20]. These differences indicate that reindeer 
366 forelimbs have stable toe joints. Measurements obtained using a linked segment model and 367 spring coefficients from a spring model demonstrated that the stiffness of goat limbs was twice 368 that of dog limbs during different activities, suggesting that goats have adapted to a rougher and 369 steeper terrain [52]. Wrist joint $d$ flexion produced a net flexion moment, which generated 370 propulsion in the middle stance phase (45-75\%) [53]. In the last $20 \%$ of the stance phase, the

371 long digital flexor tendons at interphalangeal joint $b$ and MCP joint $c$ recoiled, and joint 372 plantarflexion produced a net flexion moment; however, the net flexion moment and propulsion 373 were small (Fig. 7). Wrist joint $d$ changed between 0 and $75 \%$ of the stance phase $\left(\sim 5^{\circ}\right)$. While 374 trotting, the change trend of wrist joint $d$ in horses was similar to reindeer. The wrist joint angle 375 of horses was maintained at $180-190^{\circ}$ within $0-60 \%$ of the stance phase, then gradually 376 decreased [20]. Wrist joint $d$ in reindeer was maintained and stable over time. Reportedly, horse 377 knees during the stance phase produce a net flexion moment and the flexor muscles assist foot 378 movement, wherein the extensor muscles stabilize the joints [50]. Similarly, reindeer wrist joint $379 d$ displayed this stabilizing ability during the early and middle stance phases (0-75\%). The small 380 change in the joint angle $\left(\sim 5^{\circ}\right)$ indicated that the wrist joint plays a role in stabilizing foot 381 locomotion. Thus, the low flexibility and high stability of the forelimb joints may be beneficial 382 during long distance migrations.

\section{MCP joints as energy storage devices}

384 The MCP joints of most animals elastically store and generate energy because they are mainly 385 composed of small muscles, short pinnate muscle fibers, and long tendons $[8,54,55]$. Ligaments 386 have a protective effect on joints [56], and tendons also provide an energy advantage in high387 speed locomotion [50].

388 Reportedly, the relatively short muscle fibers and long tendons in turkey hindlimbs act like 389 springs [57], as the short muscle fibers contribute to more economical muscular energy and 390 stretching of the long tendons allows muscle fibers to generate energy with little change in length, 391 thus decreasing metabolic costs [58,59]. The MCP joints in reindeer feet also absorb and 392 generate energy during different locomotor activities (Table 1), which are manifested by an 393 elastic system for energy storage and generation (Fig. 7). The distal joints in horse forelimbs 394 recover $40 \%$ of the energy during the stance phase [41]. Furthermore, $70-80 \%$ of the plantar 395 flexors stretched at the metatarsal joints during the stance phase. The Achilles tendons, long 396 plantarflexion tendons, and plantar connective tissues of the feet absorb energy and convert it 
397 into elastic potential [39]. Reindeer MCP joints at the same position in the forelimbs also 398 performed well in energy storage during different locomotor activities and absorbed $6.42 \times 10^{-2}$, $39919.71 \times 10^{-2}$, and $33.03 \times 10^{-2} \mathrm{~J}^{\mathrm{kg}} \mathrm{kg}^{-1}$ of energy (negative power) in walk, trot 1 , and trot 2 , 400 respectively.

401

\section{Conclusions}

403 The forelimb joint angles of reindeer $\left(\alpha_{b}, \alpha_{c}, \alpha_{d}\right)$ changed in similar patterns during different 404 locomotor stance phases. The peak vertical GRF increased as locomotor speed increased. The 405 peak vertical GRFs (normalized to body mass) in walk, trot 1 , and trot 2 were 8.95, 11.33, and 406 12.80-times the body mass, respectively.

407 During different locomotor activities, the joint angles significantly differed at the touch-down, 408 mid-stance, and lift-off moments. In the trotting gaits, the MCP joint absorbed more energy than 409 the other two joints, but in the walking gaits, it absorbed less energy than the wrist joint. Across 410 different gaits and locomotor speeds, the forelimbs adopted different locomotor strategies to 411 improve locomotor performance and save energy.

412 As reindeer speed increased, the peak joint moment and net joint power both increased. The feet 413 absorbed and generated more energy at the joints. The feet first absorbed energy, then generated 414 energy at the MCP joint during the stance phase, thus performing well in elastic energy storage. 415 In the middle stance phase (45-75\%), the feet exerted a propulsive effect during the flexion of 416 the wrist joint. In the early and middle stance phases ( $0-75 \%)$, the joint angle changed very little $417\left(\sim 5^{\circ}\right)$ and the wrist joint stabilized the feet. Clearly, during long-distance migration, forelimbs 418 play stability maintenance and energy storage roles.

419 The kinematics of hindlimb and the coordination of hindlimb and forelimb of reindeer would be 420 analyzed to study the effect of reindeer foot joint on movement in the future study.

421

\section{Acknowledgments}

423 This work was supported by National Natural Science Foundation of China (No. 51675221), the 424 Science and Technology Development Planning Project of Jilin Province of China (No. 425 20180101077JC) and the Science and Technology Research Project in the 13th Five-Year Period 426 of Education Department of Jilin Province (No. JJKH20190134KJ). 


\section{References}

429 [1] Heglund N C M A , Fedak T C R and Cavagna G A . Energetics and mechanics of terrestrial 430

431

432 [2] Taylor C R, Schmidtnielsen K, Raab J L . Scaling of energetic cost of running to body size 433

434 [3] Cavagna G A, Saibene F P , Margaria R . Mechanical work in running.[J]. Journal of 435

436 [4] Roberts $\mathrm{T} \mathrm{J}$. Muscular force in running turkeys: the economy of minimizing work.[J]. 437

441 [6] Bobbert M F , Alvarez C B G , Van Weeren P R, Roepstorff L , Weishaupt M A . 442 Science, 1997, 275(5303): 1113-1115.

[5] Biewener A A . Muscle-tendon stresses and elastic energy storage during locomotion in the horse.[J]. Comparative biochemistry and physiology. Part B, Biochemistry \& molecular biology, 1998, 120(1): 73-87.

452 [10] Kim J , Rietdyk S , Breur G J . Comparison of two-dimensional and three-dimensional Validation of vertical ground reaction forces on individual limbs calculated from kinematics of horse locomotion[J]. Journal of Experimental Biology, 2007, 210(11):1885-1896.

[7] Batson E L , Paramour R J , Smith T J , Birch H L , Patterson-Kane J C, Goodship A E . Are the material properties and matrix composition of equine flexor and extensor tendons determined by their functions?[J]. Equine Veterinary Journal, 2003, 35(3): 314-318.

455 [11] Pandy M G , Kumar V , Berme N, Waldron K J . The Dynamics of Quadrupedal 456

457 [12] Giovanna G, Ivanenko Y, Lacquaniti F . A kinematic synergy for terrestrial locomotion 458 shared by mammals and birds.[J]. eLife, 2018, 7, 1-28.

459 [13] Nanua P , Waldron K J . Energy Comparison Between Trot, Bound, and Gallop Using a 460 Simple Model[J]. Journal of Biomechanical Engineering, 1995, 117(4):466-473.

461 [14] Alexander R M , Jayes A S . A dynamic similarity hypothesis for the gaits of quadrupedal 462 mammals[J]. Proceedings of the Zoological Society of London, 1983, 201(1):135-152.

463 [15] Farley C , Taylor C . A mechanical trigger for the trot-gallop transition in horses[J]. Science, 464 1991, 253(5017):306-308. 
465 [16] Hildebrand M. Analysis of Asymmetrical Gaits[J]. Journal of Mammalogy, 1977(2):131466 156.

467 [17] Hildebrand M. The quadrupedal gaits of vertebrates[J]. BioScience, 1989(39):766-775.

468 [18] Hoyt D F , Taylor C R . Gait and the energetics of locomotion in horses[J]. Nature, 1981, 469 292(5820):239-240.

470 [19] Minetti A E , Ardigo L P , Reinach E , Saibene F . The relationship between mechanical 471 work and energy expenditure of locomotion in horses[J]. Journal of Experimental Biology,

472 1999, 202(17):2329-2338.

473 [20] Dutto D J , Hoyt D F , Clayton H M , Cogger E A , Wickler S J . Joint work and power for

474

475 both the forelimb and hindlimb during trotting in the horse[J]. Journal of Experimental Biology, 2006, 209(20):3990-3999.

476 [21] Dutto D J , Hoyt D F , Cogger E A, Wickler S J . Ground reaction forces in horses trotting 477

478 up an incline and on the level over a range of speeds. Journal of Experimental Biology, 2004,

479 [22] Riemersma D J , Schamhardt H C , Lammertink J L M A . In vivo tendon load and tendon 480 strain in the horse[J]. Journal of Biomechanics, 1985, 18(7):556.

481 [23] Rumph P F , Lander J E , Kincaid S A , Baird D K , Kammermann J R , Visco D M . 482 Ground reaction force profiles from force platform gait analyses of clinically normal mesomorphic dogs at the trot[J]. American Journal of Veterinary Research, 1994, 55(6):756761.

485 [24] Witte T H . Determination of peak vertical ground reaction force from duty factor in the 486 horse (Equus caballus)[J]. Journal of Experimental Biology, 2004, 207(21):3639-3648.

487 [25] Dutto D J . Moments and power generated by the horse (Equus caballus) hind limb during 488 jumping[J]. Journal of Experimental Biology, 2004, 207(4):667-674.

489 [26] Merkens H W, Schamhardt H C , Osch G J V M V , BOGERT A J . Ground reaction force 490 491 patterns of Dutch Warmblood horses at normal trot[J]. Equine Veterinary Journal, 1993, 25(2):134-137.

492 [27] Tol P P J V D , Metz J H M, Noordhuizen-Stassen E N , Back W, Braam C R, Weijs W A . The Vertical Ground Reaction Force and the Pressure Distribution on the Claws of Dairy Cows While Walking on a Flat Substrate[J]. Journal of dairy science, 2003, 86(9):2875-2883.

495 [28] Vereecke E, D'Août K, Elsacker L V , De Clercq D , Aerts P. Functional analysis of the gibbon foot during terrestrial bipedal walking: Plantar pressure distributions and threedimensional ground reaction forces[J]. American Journal of Physical Anthropology, 2005,

499 [29] Zhang R, Li G Y, Pan R D, Wang Q, Li J Q . Structure, Morphology and Composition of 500 Fur on Different Parts of Reindeer (Rangifer Tarandus) Foot[J]. Micron, 2019, 126, 1-8.

501 [30] Wareing K, Tickle P G, Stokkan K A, Codd J R , Sellers W I . The musculoskeletal 502 503 anatomy of the reindeer (Rangifer tarandus): fore- and hindlimb[J]. Polar Biology, 2011, 34(10):1571-1578. 
504 [31] Fancy S G , Pank L F, Whitten K R, Regelin W L . Seasonal movements of caribou in

505 arctic Alaska as determined by satellite[J]. Rangifer, 2010, 10(3):644-650.

506 [32] Essén-Gustavsson B, Rehbinder C . Skeletal muscle characteristics of reindeer (Rangifer

507 tarandus L.)[J]. Comp Biochem Physiol. A Comp Physiol, 1985, 82(3):675-679.

508 [33] Zhang R, Qiao Y, Ji Q L, Ma S S , Li J Q . Macro-microscopic research in reideer

509

510

$511[34]$

512

513

514 [35] Roberts T J . Adjusting muscle function to demand: joint work during acceleration in wild 515

516 [36] Vereecke E E , Aerts P. The mechanics of the gibbon foot and its potential for elastic 517

518

519 [37] Biewener A. Scaling body support in mammals: limb posture and muscle mechanics[J]. 520

$521[38]$

522

523

524 [39] Vereecke E E , D’Août K, Payne R, Aerts P. Functional analysis of the foot and ankle

525

526 [40] Harrison S M, Whitton R C , Kawcak C E, Stover S , Pandy M G . Relationship between 527

528

529 [41] Biewener A A, Thomason J J , Lanyon L E. Mechanics of locomotion and jumping in the 530

531

$532[42]$

533

534 [43] Mcguigan M P, Yoo E, Lee D V, Biewener A A . Dynamics of goat distal hind limb 535

536

$537[44]$ 538

539 [45] Full R J , Koditschek D E . Templates and anchors: neuromechanical hypotheses of legged 540

541 [46] Geyer H, Seyfarth A, Blickhan R. Compliant leg behaviour explains basic dynamics of 542 543 horse (Equus): in vivo stress in the tibia and metatarsus[J]. Proceedings of the Zoological Society of London, 1988, 214(3):547-565. muscle-tendon function in response to locomotor grade $[\mathrm{J}]$. Journal of Experimental Biology, 2009, 212(13):2092-2104.

44] Donelan J M , Kram R, Kuo A D . Simultaneous positive and negative external mechanical work in human walking[J]. Journal of Biomechanics, 2002, 35(1):117-124. locomotion on land[J]. Journal of Experimental Biology, 1999, 202(Pt 23):3325-3332. walking and running[J]. Proceedings of the Royal Society B: Biological Sciences, 2006, 273(1603):2861-2867. 
544 [47] Farley C T , Glasheen J , Mcmahon T A . Running springs: Speed and animal size[J]. 545 Journal of Experimental Biology, 1994, 185(1):71-86.

546 [48] Cavagna G A, Thys H , Zamboni A. The sources of external work in level walking and 547 running[J]. Journal of Physiology, 1976, 262(3):639-657.

548 [49] Muir G D , Gosline J M , Steeves J D . Ontogeny of bipedal locomotion: walking and 549 running in the chick[J]. Journal of Physiology, 1996, 493(2):589-601.

550 [50] Schaller N U , D"Aout K, Villa R, Herkner B , Aerts P . Toe function and dynamic 551 pressure distribution in ostrich locomotion[J]. Journal of Experimental Biology, 2011, 552 214(7):1123-1130.

553 [51] Hildebrand M . Analysis of tetrapod gaits: general considerations and symmetrical 554 gaits[J]. Advances in Behavioral Biology, 1976, 18: 203-236.

555 [52] Lee D V , Isaacs M R, Higgins T E, Biewener A A , Mcgowan C P. Scaling of the Spring 556

557 in the Leg during Bouncing Gaits of Mammals[J]. Integrative and Comparative Biology, 2014, 54(6):1099-1108.

558 [53] Ishida H. Kinesiological aspects of bipedal walking in gibbons[J]. The Lesser ape, 1984: $559 \quad 135-145$.

560 [54] Payne R C , Hutchinson J R , Robilliard J J , Smith N C, Wilson A M . Functional 561

562 specialisation of pelvic limb anatomy in horses (Equus caballus)[J]. Journal of Anatomy, 2005, 206(6):557-574.

563 [55] Wickler, S. J . In vivo muscle function vs speed II. Muscle function trotting up an incline[J]. 564 Journal of Experimental Biology, 2005, 208(6):1191-1200.

565 [56] Schaller N U, Herkner B , Villa R, Aerts P . The intertarsal joint of the ostrich (Struthio 566 camelus): Anatomical examination and function of passive structures in locomotion[J]. 567 Journal of Anatomy, 2009, 214(6):830-847.

568 [57] Ker R F , Alexander R M , Bennett M B . Why are mammalian tendons so thick?[J]. Journal 569 of Zoology, 1988, 216(2):309-324.

570 [58] Rall J A . Energetic Aspects of Skeletal Muscle Contraction[J]. Exercise and Sport Sciences 571 Reviews, 1985, 13(1):33-74.

572 [59] Biewener A A , Roberts T J . Muscle and tendon contributions to force, work, and elastic 573 energy savings: A comparative perspective[J]. Exercise and Sport Sciences Reviews, 2000, 574 28(3):99-107. 
Figure 1

Schematic diagram of the test site .

The red line indicates the data acquisition area $\left(3 \times 1.5 \mathrm{~m}^{2}\right)$, the yellow line indicates the runaway (77 m long), and the green circles indicate the rest areas. A fence (1.5 m high) was set around all areas.

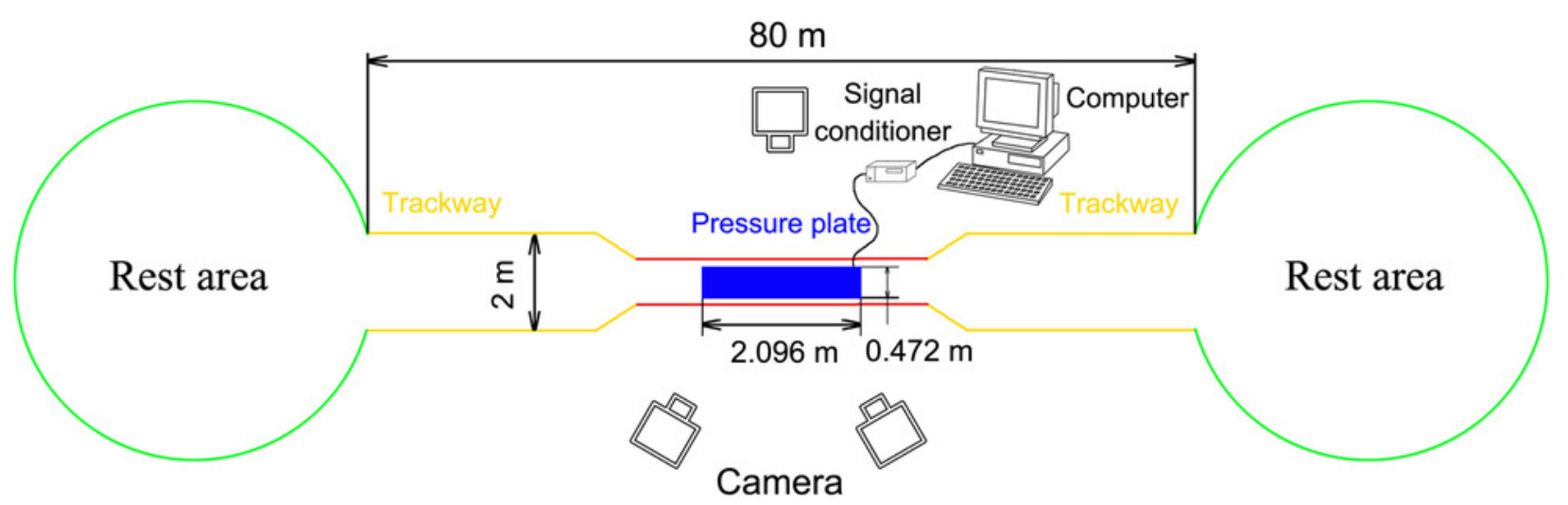




\section{Figure 2}

Reindeer locomotor gait, position of right forelimb joint, and schematic diagram used to calculate net moment and power.

A. Markers on the reindeer right forelimb $(a, b, c, d, e)$. B. Distinguish between between reindeer walking and trotting gait. RF, RH, LF and $\mathrm{LH}$ are right forefoot, right hindfoot, left forefoot and left hindfoot, respectively. C. Three-dimensional model of the limb based on CT scan image reconstruction of the adult male reindeer right forelimb to determine the locations of the markers $(a, b, c, d)$. D. Reindeer limb model for calculating the net joint moment $M_{\mathrm{m}}$. Net joint moment is the product of the GRF and force arm L. $L$ is the vertical distance from the center of rotation of the joint to the GRF. The arrow indicates that the muscle produced a positive net moment direction at the joint. E. Reindeer limb model for calculating the net joint power $P_{\mathrm{m}}$. The angular velocity of the joint was calculated from the derivative of the angle $\left(\alpha_{b}, \alpha_{c}, \alpha_{d}\right)$ that changed over time. The arrow indicates the positive direction of the angular velocity. This model is the state of the reindeer right forelimb in the $25 \%$ of the stance phase. Definition: $a$. the dorsal hoof of the third digit; $b$. the joints of the proximal phalanx and middle phalanx of the third digit (interphalangeal joint); c. the joint of the proximal phalanx of the digit and metacarpal (metacarpophalangeal joint); $d$. wrist joint; e. elbow joint; I. the proximal phalanx; II. the middle phalanx; III. the distal phalanx. 


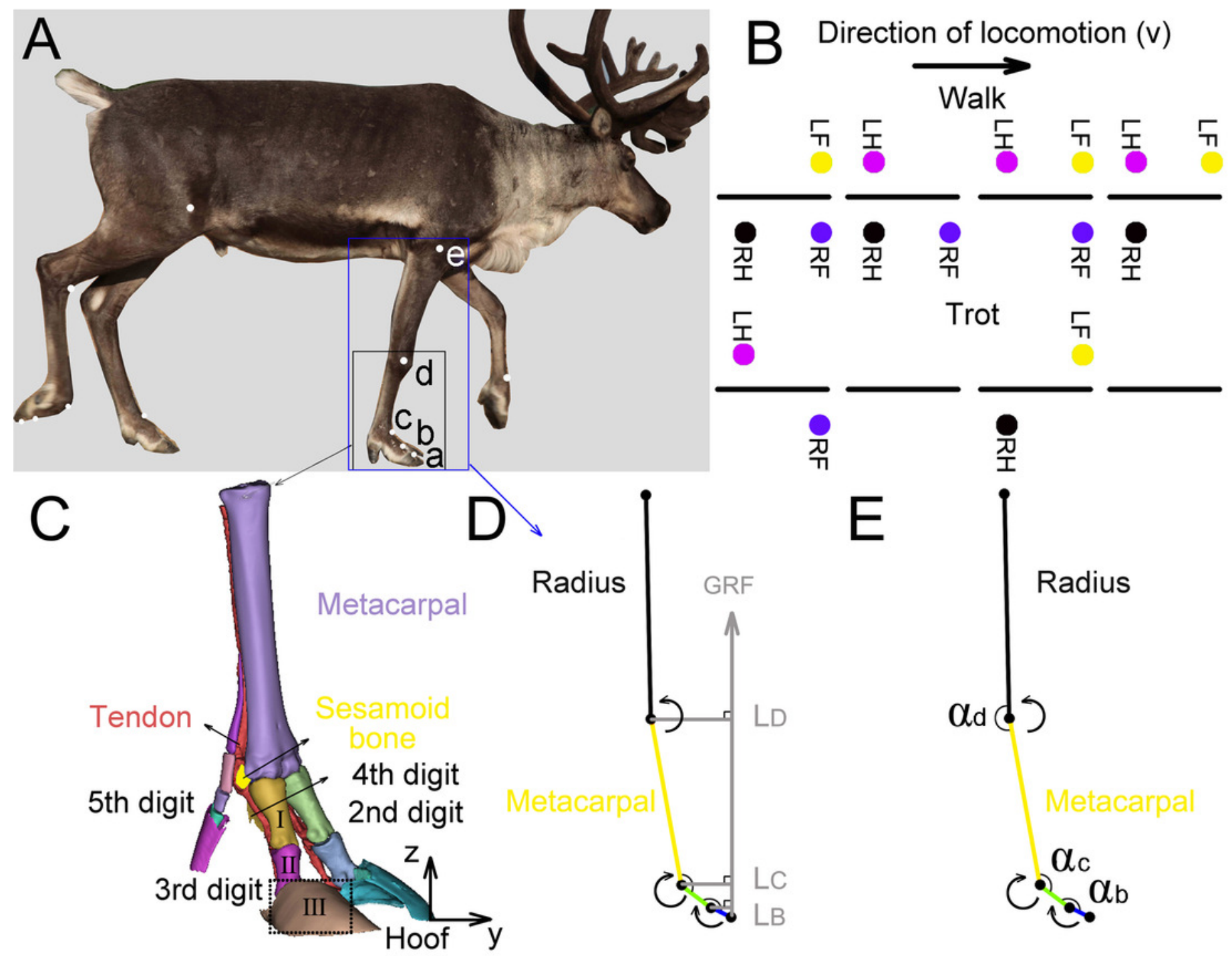


Figure 3

Locomotor state of the reindeer right forelimb in the stance phase.

A. Walk. B. Trot 1. C. Trot 2. From left to right: moment of touch-down, $25 \%$ of the stance phase, mid-stance, $75 \%$ of the stance phase, and lift-off.
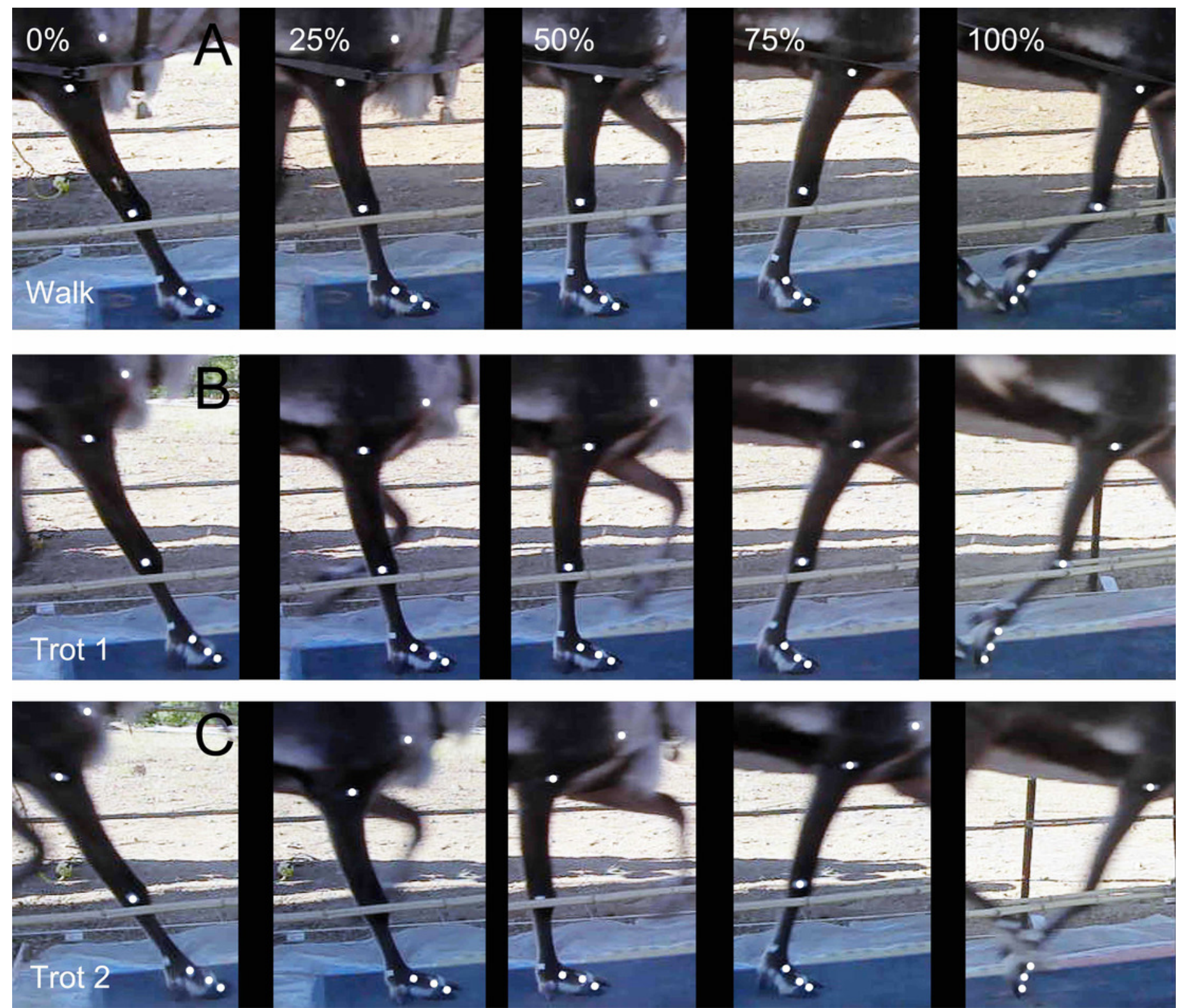


\section{Figure 4}

The means and standard deviations of the joint angles $\left(\alpha_{b}, \alpha_{c}, \alpha_{d}\right)$ from the four samples.

A, B, C: Curve of joint angle $\alpha_{b}$ during the stance phase at walk, trot 1 , and trot 2 ,

respectively. D, E, F: Curve of joint angle $\alpha_{c}$ during the stance phase at walk, trot 1 , and trot

2, respectively. G, H, I: Curve of joint angle $\alpha_{d}$ during the stance phase at walk, trot 1 , and

trot 2, respectively. J, K, L: Motion of the right forelimbs during the stance phase at walk, trot 1 , and trot 2 , respectively. Yellow, green, blue, and pink lines represent reindeer samples 1 , 2,3 , and 4 , respectively. 


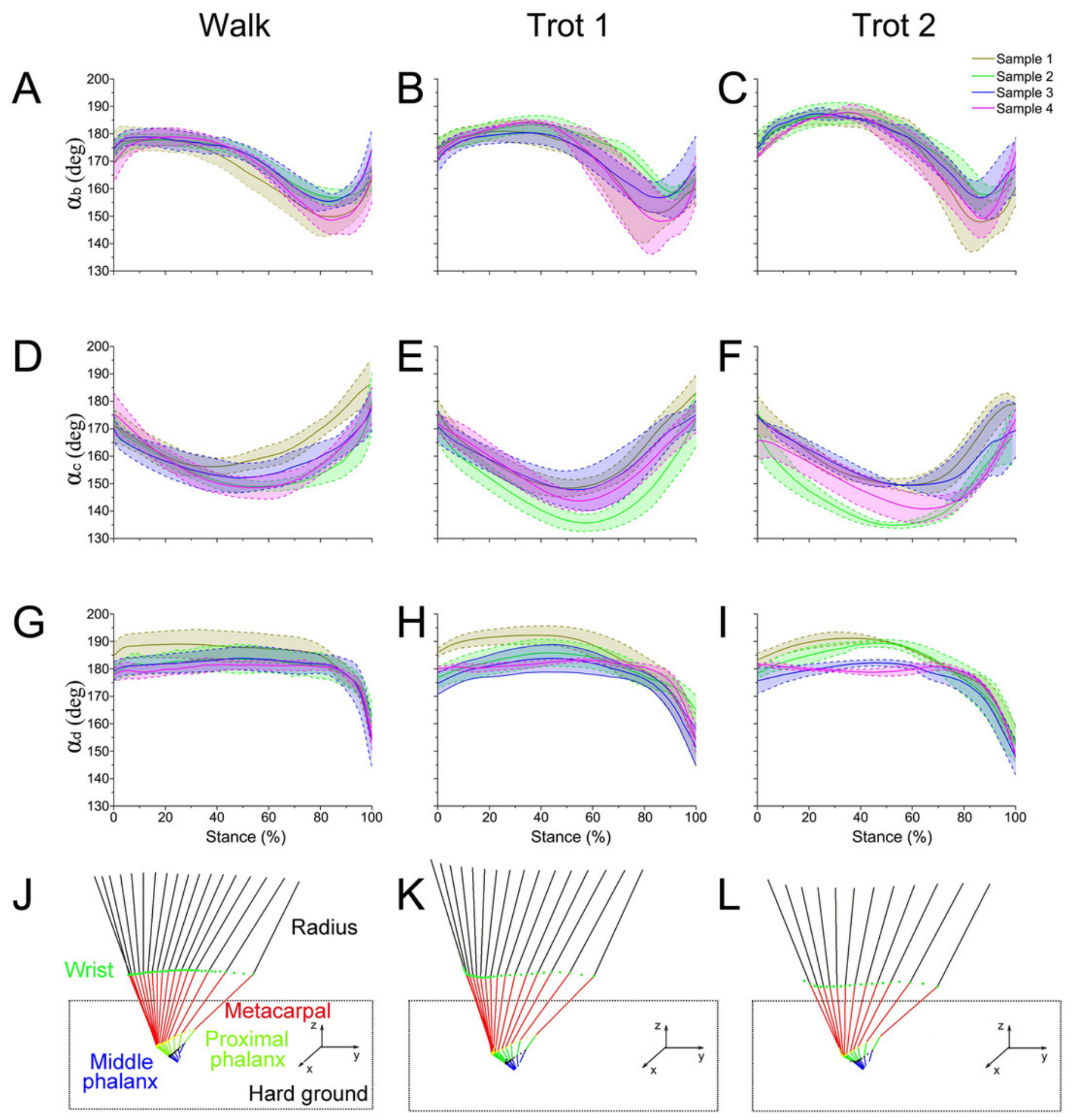




\section{Figure 5}

Means and standard deviations of the three joint angles in walk, trot 1, and trot 2 locomotion on hard ground during the stance phases.

A. Curve of joint angle $\alpha_{b}$ over time. B. Curve of joint angle $\alpha_{c}$ over time. C. Curve of joint angle $\alpha_{d}$ over time. The yellow, pink, and blue lines represent walk, trot 1 , and trot 2 , respectively.
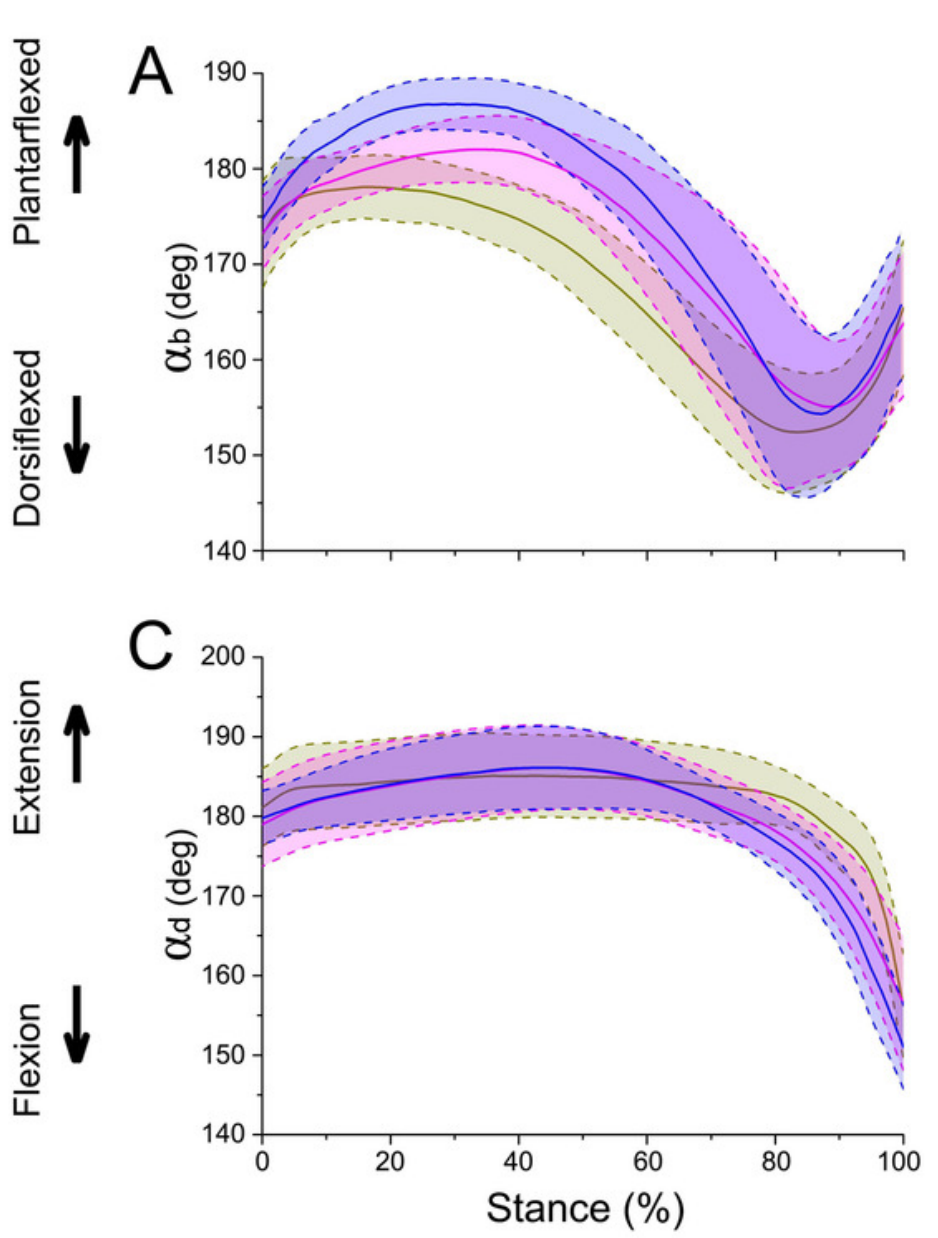

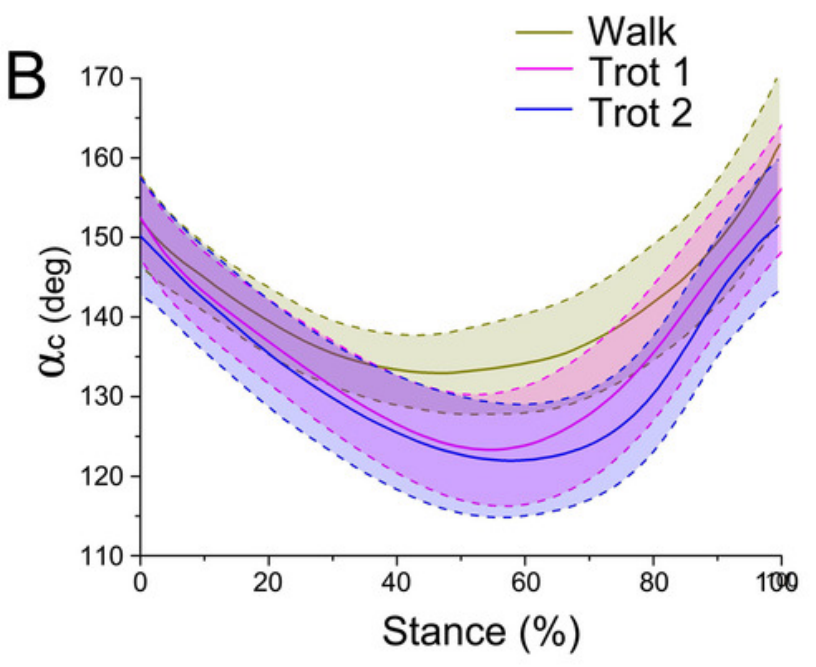


Figure 6

Means and standard deviations of the vertical GRFs (normalized to body mass) of the right forelimbs on hard ground in walk $(A)$, trot $1(B)$, and trot $2(C)$ during the stance phase.

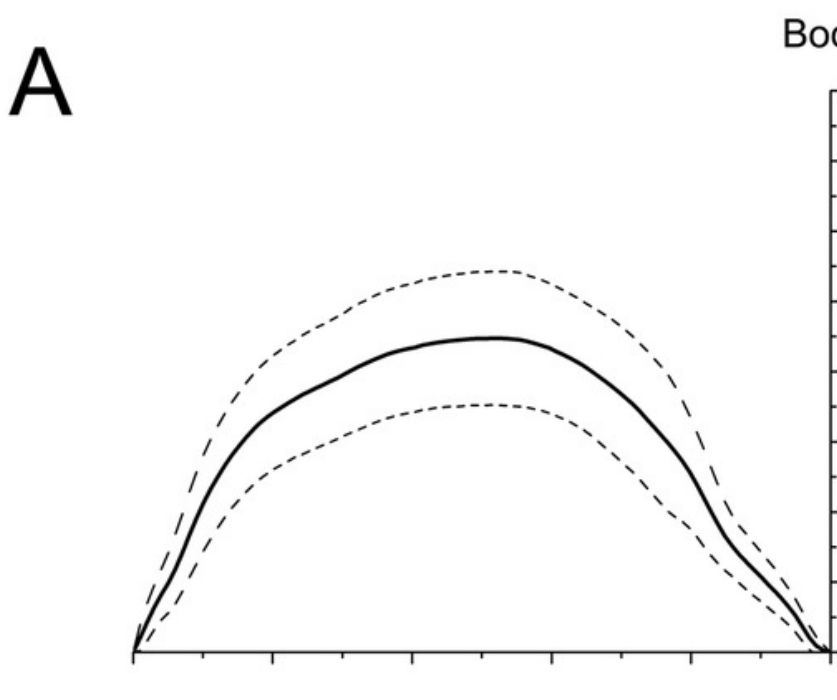

Body mass
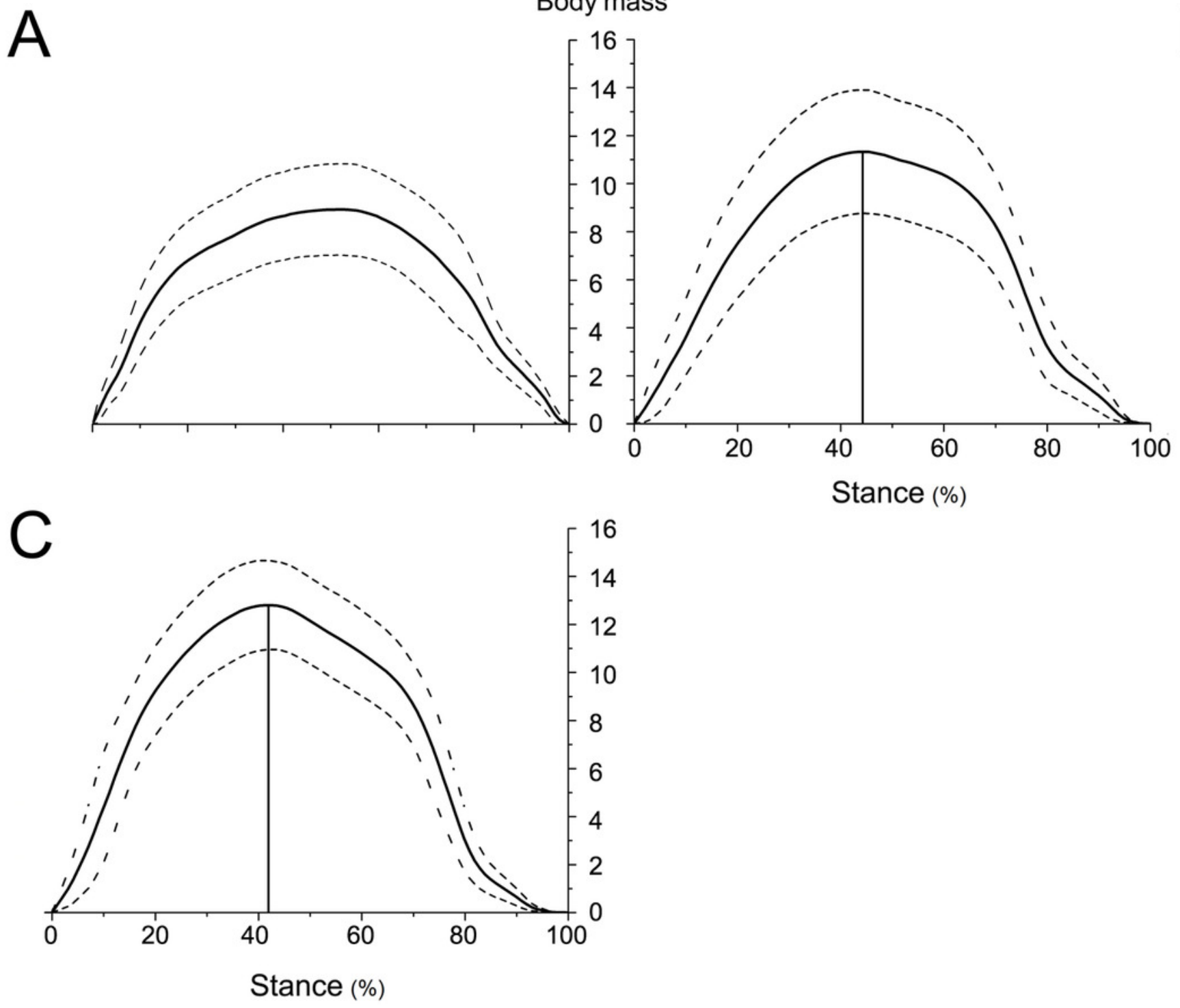


\section{Figure 7}

Means and standard deviations of net joint moments (yellow solid line, mean; yellow shaded area, standard deviation) and net joint power (pink solid line, mean; pink shaded area, standard deviation) of the right forelimb joints.

A, B, C: The net joint moment and net joint power of interphalangeal joint $b$ in walk, trot 1 , and trot 2. D, E, F: The net joint moment and net joint power of MCP joint $c$ in walk, trot 1 , and trot 2. Interphalangeal joint $b$ and MCP joint $c$ are indicated by the left and right circles, respectively. 

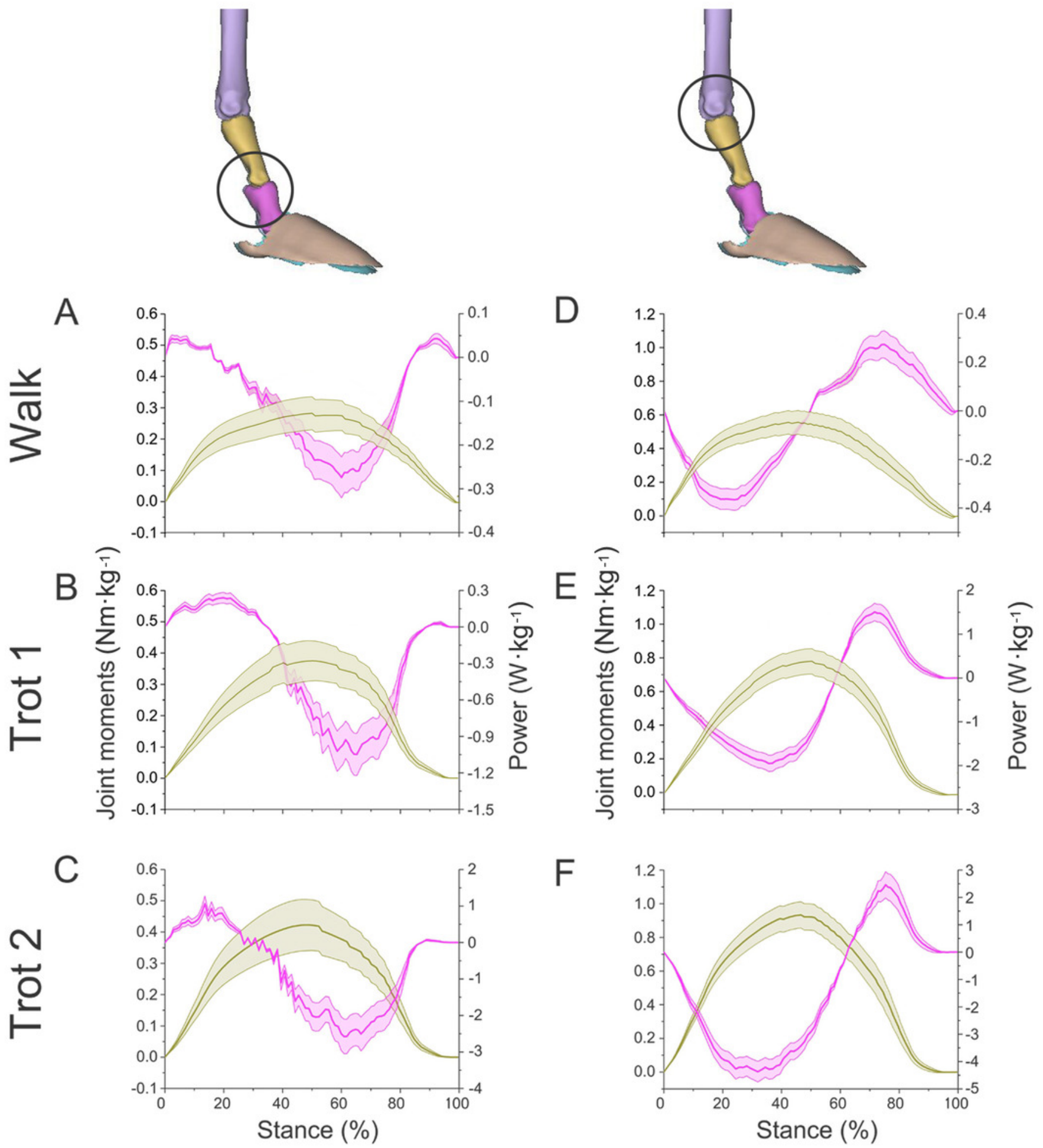


\section{Figure 8}

Means and standard deviations of net joint moment (yellow solid line, mean; yellow shaded area, standard deviation) and net joint power (pink solid line, mean; pink shaded area, standard deviation) at wrist joint $d$ of the right forelimb.

A, B, C: The net joint moment and net joint power of wrist joint $d$ in walk, trot 1 , and trot 2 , respectively. 

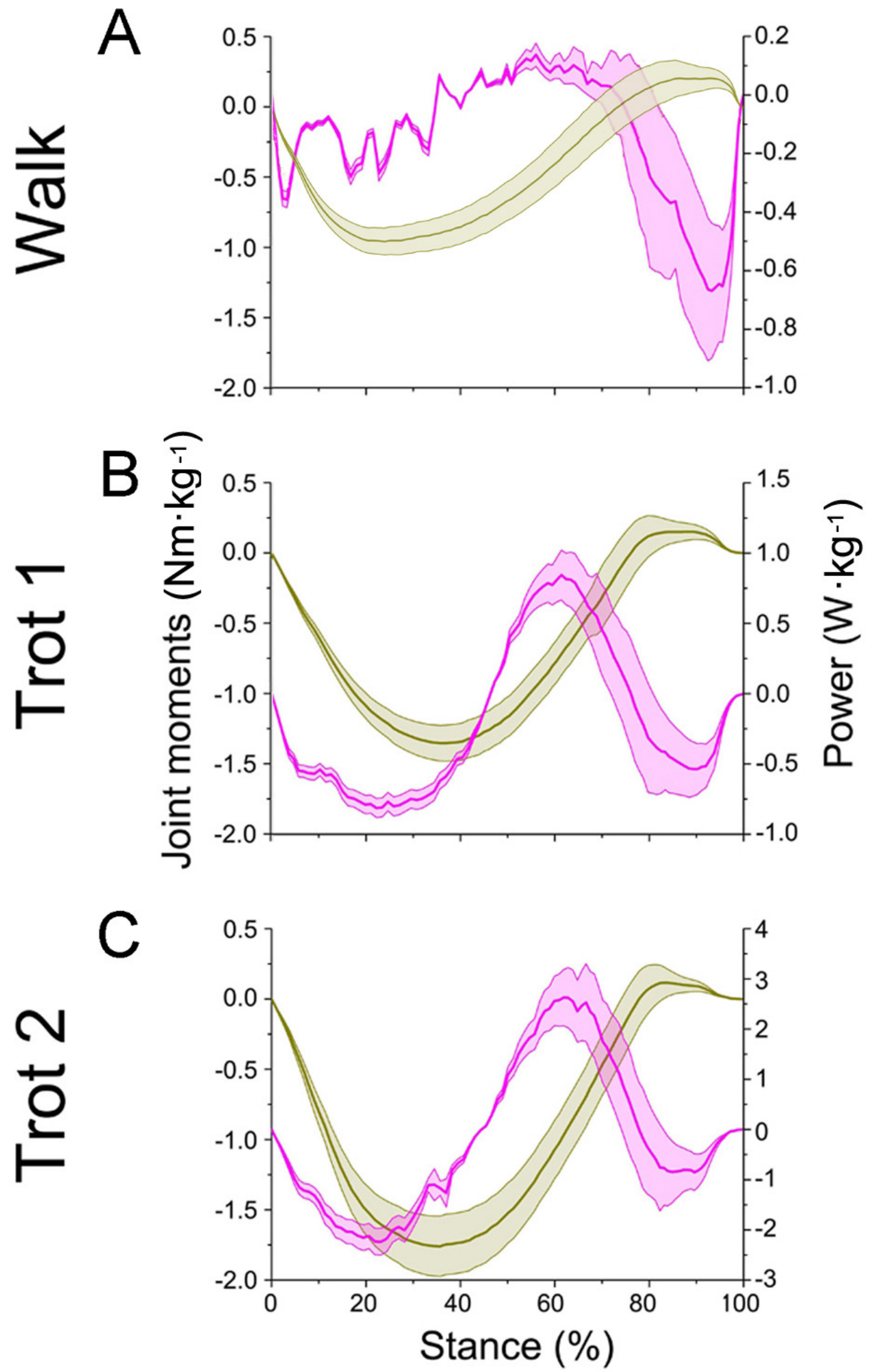


\section{Figure 9}

Numerical statistical analysis of the effect of locomotor gait and speed to the forelimb joint angles $\left(\alpha_{b}, \alpha_{c}, \alpha_{d}\right)$ of the reindeer at the touch-down (0\%), mid-stance (50\%), and lift-off (100\%).

A, C, E: Analysis of the significant effects of walking and trotting gait on joint angles $\alpha_{b}, \alpha_{c}$ and $\alpha_{\mathrm{d}}$. B, D, F: Analysis of the significant effects of the locomotor speed of trotting gaits on joint angle $\alpha_{\mathrm{b}}, \alpha_{\mathrm{c}}$, and $\alpha_{\mathrm{d}} * P<0.05$. 

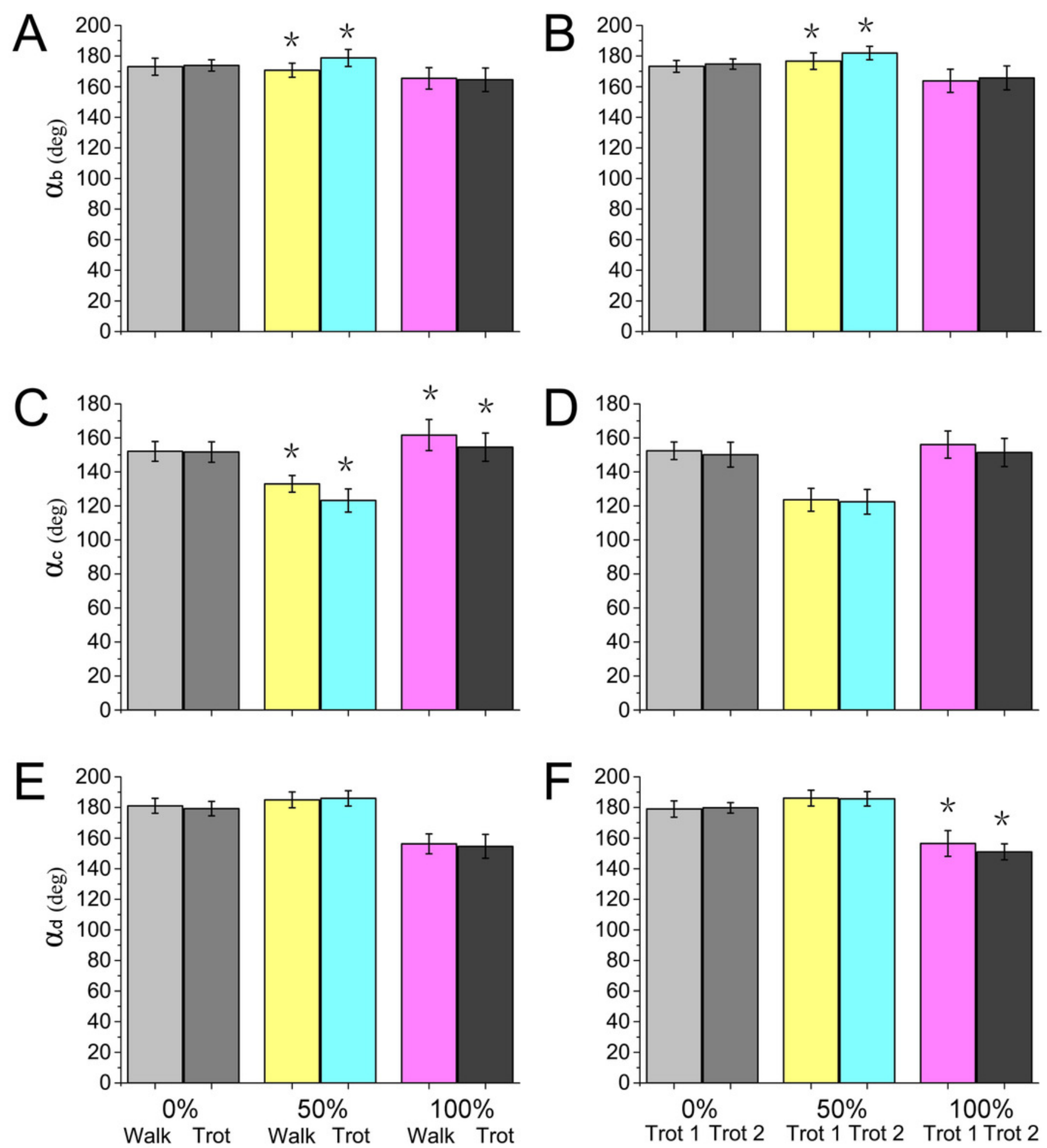


\section{Table $\mathbf{1}$ (on next page)}

Positive, negative, and net power produced by reindeer forelimbs joints during different locomotion activities. 


\begin{tabular}{ccccc}
\hline Joint & Locomotion & Positive power $\left(\mathrm{J} \cdot \mathrm{kg}^{-1}\right)$ & Negative power $\left(\mathrm{J} \cdot \mathrm{kg}^{-1}\right)$ & Net power $\left(\mathrm{J} \cdot \mathrm{kg}^{-1}\right)$ \\
\hline & Walk & $5.84 \times 10^{-3}$ & $-6.09 \times 10^{-2}$ & $-5.91 \times 10^{-2}$ \\
\hline $\mathrm{b}$ & Trot 1 & $1.50 \times 10^{-2}$ & $-10.63 \times 10^{-2}$ & $-8.97 \times 10^{-2}$ \\
& Trot 2 & $2.95 \times 10^{-2}$ & $-18.79 \times 10^{-2}$ & $-14.50 \times 10^{-2}$ \\
& Walk & $4.64 \times 10^{-2}$ & $-6.42 \times 10^{-2}$ & $-2.74 \times 10^{-2}$ \\
& Trot 1 & $9.58 \times 10^{-2}$ & $-19.71 \times 10^{-2}$ & $-15.00 \times 10^{-2}$ \\
& Trot 2 & $9.52 \times 10^{-2}$ & $-33.03 \times 10^{-2}$ & $-29.50 \times 10^{-2}$ \\
& Walk & $1.39 \times 10^{-2}$ & $-10.69 \times 10^{-2}$ & $-8.77 \times 10^{-2}$ \\
& Trot 1 & $5.00 \times 10^{-2}$ & $-9.98 \times 10^{-2}$ & $-6.44 \times 10^{-2}$ \\
& Trot 2 & $12.02 \times 10^{-2}$ & $-15.09 \times 10^{-2}$ & $-4.80 \times 10^{-2}$ \\
\hline
\end{tabular}

1 